Review Article

\title{
The Effect of COVID- I 9 Pandemic on Immunization Services in India - Possible Challenges and way forward
}

\author{
Varun Singh
}

B.Pharm, MBA (Healthcare Management), Public Health Expert.

Former Consultant (Urban Health and RCH Division), Ministry of Health and Family Welfare (Government of India), New Delhi. DOI: https://doi.org/10.24321/2455.7048.202018

\section{I $\quad \mathbf{N} \quad \mathbf{F} \quad \mathbf{O}$}

E-mail Id:

varunsingh.mohfw@gmail.com

Orcid Id:

https://orcid.org/0000-0003-0264-1576

How to cite this article:

Singh V. The Effect of COVID-19 Pandemic on Immunization Services in India - Possible Challenges and way forward. Epidem Int 2020; $5(2): 53-60$.

Date of Submission: 2020-06-01

Date of Acceptance: 2020-06-o9

\section{$\begin{array}{llllllll}\mathbf{A} & \mathbf{B} & \mathbf{S} & \mathbf{T} & \mathbf{R} & \mathbf{A} & \mathbf{C} & \mathbf{T}\end{array}$}

\begin{abstract}
The novel disease COVID-19 which has affected more than 200 countries globally including India, has been declared as pandemic by World Health Organization. The Government of India (GOI) has taken timely measures to contain the human-to-human transmission of COVID-19 in the Country through implementation of lockdown to restrict the travel with relaxation for availing essential commodities and medical services. Domestic and International transport facilities have been stopped. The expansion of this pandemic has adversely affected health seeking behavior of community for other health conditions and the service delivery of essential health services including immunization. Routine immunization targets to immunize around 157 million beneficiaries each year against twelve vaccine preventable diseases. However, due to lockdown and further strategy of containment of COVID - 19, the service delivery under Universal Immunization Program (UIP) is badly affected. In order to avert the implication of delayed immunization on future of the India's immunization program, this gap need to be addressed by taking timely measures for immunization of due beneficiaries. The present article is an attempt to explain existing immunization practices in India, enumerate possible challenges in delivering immunization services due to COVID-19 and enlist suggestive strategies to overcome those challenges.
\end{abstract}

Keywords: COVID-19, Universal Immunization Program, UIP, Pandemic, Vaccine Preventable Disease, VPD, Service Delivery

\section{Introduction}

COVID-19 is the infectious disease caused by novel strain of coronavirus (SARS-CoV-2) reported first in Wuhan, China, in December 2019. Coronaviruses are a family of viruses that causes illness like common cold, MERS (Middle East Respiratory Syndrome), SARS (Severe Acute Respiratory Syndrome) etc. The coronaviruses typically cause respiratory symptoms. The disease can spread from human to human through small droplets from the nose or mouth which can spread when a person with COVID-19 coughs or exhales and other human comes in contact with droplet either directly or through objects.

As on $6^{\text {th }}$ June 2020, the COVID-19 Pandemic has affected more than 200 countries worldwide, with confirmed 6.94 
million cases and 0.40 million deaths. India reported its first case of COVID-19 in Kerala on 30th January 2020, and until $6^{\text {th }}$ June 2020, India has reported 246622 cases with aprox. 6946 deaths. ${ }^{1}$

The Government of India has taken several timely measures to restrict the entry of SARS-CoV-2 in the community and to interrupt its transmission. Since $3^{\text {rd }}$ week of March, Gol has restricted entry of travelers in India from abroad, provisions have been put in place to quarantine passengers for a minimum of 14 days. Government had also undertook evacuation operations to bring back Indian from other countries. Inter-state transport facilities have also been facilitated for domestic workers, labours and students those were stuck in another city away from home. The nationwide lockdown with permission for availing essentials goods and medical services is being implemented since $22^{\text {nd }}$ March 2020 i.e. the date of Janata Curfew till date. It is also appreciated that various preventive measures such as use of mask, social distancing and quarantine have been strictly followed. However, during lockdown 3.0 Ministry of Health and Family Welfare (MoHFW) has done risk profiling of all districts of the country into Red, Green and Orange Zones on the basis of number of confirmed cases to provide few relaxation to bring social and economic activities on track.

- Green Zones: Districts with either zero confirmed cases till date; or, no confirmed case in the last 21 days.

- Red Zones: Classification of district will take into account the total number of active cases, doubling rate of confirmed cases, extent of testing and surveillance feedback from the districts.

- Orange Zone: Those districts, which are neither defined as Red nor Green.

The zone-wise classification of districts is a dynamic process, MoHFW is updating States/ UTs on a weekly basis, or earlier, as required. While States and UTs can include additional districts as Red and Orange Zones. ${ }^{2}$ There are specific and well defined relaxation for public in each classified zone as well. The Gol also announced lockdown phase 4.0 and subsequent phase 5.0 , ending on $30^{\text {th }}$ June 2020 with more relaxation to public to carryout economic activities. MoHFW has taken into account the criteria of 'Containment \& Buffer Zone' and 'areas beyond Buffer Zone \& Green Zone' for delivery of essential health services. The Containment zones are defined as an area where COVID-19 cases are reported. Surrounding areas of containment zone with risk for COVID-19 spread are called as buffer zone. Beyond buffer and green zone are areas having limited/ no risk of pandemic.

Government of India has always been striving towards reducing mortality and morbidity due to Vaccine Preventable Diseases (VPD). Routine Immunization (RI) activities under Universal Immunization Programme (UIP) targets to vaccinate 27 million newborns each year with all primary doses and around 100 million children from 1 to 5 years of age with booster doses of UIP vaccines. In addition, 30 million pregnant mothers are targeted for Tetanus toxoid (TT)/Tetanus and adult Diphtheria (Td) vaccination each year. In order to vaccinate this cohort of 157 million beneficiaries, around 10 million immunization sessions are conducted each year, majorly at the village level. Additionally, Special Immunization campaign Activities (SIA) to reach missed \& drop-out children during immunization session, are carried-out. These activities act as a catalyst in boosting up immunization coverage in a well-defined geographical area and/or areas having low immunization coverage. It supplements routine immunization activities to reach set goal of immunization coverage. The potent and safe vaccines delivery to children is ensured through a network of around 27,000 functional cold chain points across the country wherein vaccines are stored at recommended temperatures. The monitoring of these are performed by Real-time mobile based application eVIN (Electronic Vaccine Intelligence Network) which provides real-time information about the stock position and cold chain equipment temperature. Measures have also been adopted to speed-up the processes of Adverse Events Following Immunization (AEFI) recording, reporting and investigation as well. Since 2014, many new vaccines have been introduced under UIP, which includes Rotavirus vaccine, Japanese Encephalitis (JE) vaccine for adults, Inactivated Poliovirus Vaccine (IPV); Measles Rubella vaccine (MR) and, Pneumococcal Conjugate Vaccine (PCV). Tetanus Toxoid (TT) is also replaced with Tetanus and adult Diphtheria (Td) vaccine in 2019. ${ }^{3}$

As on date, UIP provide free of cost immunization to pregnant women against tetanus and, to children and adolescents against twelve vaccine preventable diseases i.e. Diphtheria, Pertussis, Tetanus, Polio, Measles, Rubella, Childhood Tuberculosis, Hepatitis B and Meningitis/Pneumonia caused by Hemophilus Influenza type B, Pneumococcal Pneumonia, Rotavirus diarrhea and Japanese Encephalitis (JE endemic districts only).

Full Immunization Coverage (FIC) is the percentage of one-year-olds who have received one dose of BCG vaccine, three doses of polio vaccine, three doses of pentavalent vaccine, and one dose of measles vaccine. As per NFHS-4 (2014-15), India's FIC was only $62 \%$ with intra/inter-states, urban-rural and antigen-wise variations. Socio-economic status, geographical disparities, individual's/religious beliefs, vaccine hesitancy etc. are major contributors for slow-paced achievement of FIC.

As a major step to improve the immunization coverage in India, intensified campaign named "Mission Indradhanush (MI)" was launched in December 2014 with aim to reach 90\% Full Immunization Coverage (FIC) by 2020. 
Subsequently, to accelerate vaccine coverage and participation of multiple stakeholders towards a common goal of $90 \%$ FIC, Honourable Prime Minister (PM) launched Intensified Mission Indradhanush (IMI) in October 2017. The programme continuously reviewed by Honourable Prime Minister under Pro-Active Governance and Timely Implementation (PRAGATI) review. With political priority, extensive reviews and dedicated work of healthcare staffs at each level, Mission Indradhanush covered around 33.9 million children and 8.7 million pregnant women till January $2020 .^{4}$

IMI showed an average improvement of 18.5 percentage points in the full immunization coverage in 190 IMI districts. ${ }^{5}$ Mission Indradhanush was also a part of seven leading Central Government schemes under Gram Swaraj Abhiyan (GSA) and extended GSA. The intensified mission Indradhanush 2.0 activities have carried out between December 2019 - March 2020 with aim to strengthen routine immunization services and to achieve the coverage of more than $90 \%$ immunization. ${ }^{6}$

Further, to alert and support the Country during Vaccine Preventable Disease (VPD) outbreak, India has different surveillance models and adopted various strategies to increase the robustness of the VPD surveillance system in the country such as assessment of the VPD burden, enhanced coordination between different surveillance sources, involvement of the private sector and strengthened sentinel surveillance for new antigens. Furthermore, using the existing platform of polio and measles surveillance systems, i.e. WHO's National Polio Surveillance Project (NPSP) in concurrence with the Gol designed laboratory surveillance for diphtheria, pertussis and neonatal tetanus is supported. NPSP also carries out surveillance for Acute Flaccid Paralysis (AFP) and measles in India and, provides needed technical and training support for AFP and measles surveillance. ${ }^{7,8}$ In addition, laboratory-supported VPD surveillance is an essential tool for providing real-time information about VPD cases in the Country. Laboratorysupported VPD surveillance for diphtheria, pertussis and neonatal tetanus is currently functional in States like Bihar, Haryana, Himachal Pradesh, Karnataka, Kerala, Madhya Pradesh, Punjab, and Uttar Pradesh. ${ }^{9,10}$

Government's high-level commitment to save the lives of children, adolescents and pregnant women have resulted in increased immunization coverage in last few years. Morbidity and mortality due to VPDs have immensely declined. India's achievements in Universal Immunization Programme and various initiatives such as eVIN and Mission Indradhanush have been appreciated at international forums. However, the COVID-19 pandemic has adversely affected the delivery of few essential services including UIP that need to be resumed. Present article is an attempt to chalk out challenges system may face and suggestive strategies to save our future generation from VPDs while fighting against COVID-19.

\section{Methodology}

Desk review of literatures, i.e. research articles, published guidelines, Gol press releases. The Government of India (Gol) and international website providing COVID-19 data and situation reports.

\section{Discussion}

\section{Effect on Fixed \& Outreach Session}

Immunization services to children (0-5 years) are being provided through special campaign and routine immunization sessions held at fixed sites (healthcare facilities) and outreach sites, i.e. at anganwadi centers, in villages etc. As per Coverage Evaluation Survey conducted by UNICEF in 2009 , only $9 \%$ of immunization services are delivered by private hospitals/clinics, thus, laying more responsibility on India's public health system for reaching each and every child and ensuring full and complete immunization. Tetanus-diphtheria ( $\mathrm{Td}$ ) vaccination of pregnant women and adolescents are also covered under UIP. 91\% of vaccination in India are delivered by Public health sector ${ }^{11}$ through fixed sites ( $24 \%$ at government/ municipal hospitals and $13 \%$ at $\mathrm{PHC} / \mathrm{CHC}$ ) and $54 \%$ from outreach session held at anganwadi center (26\%), sub centre (19\%) \& some places in village (9\%). During this lockdown period of more than two months, routine immunization services mainly outreach sessions have been partially or fully suspended in the states leaving large number of missed children \& dropout. The pandemic has extensively affected both fixed and outreach immunization sessions. Travel restrictions during nationwide lockdown to break the chain of COVID-19 transmission has limited movement of health workers and caregivers as well as interrupted vaccine and logistics supply.

To guide the States in delivering essential health services during the pandemic, MoHFW has released the guidelines titled "Enabling Delivery of Essential Health Services during the COVID 19 Outbreak" on $14^{\text {th }}$ April 2020. ${ }^{12}$ The Guideline has suggested $\mathrm{RMNCH}+\mathrm{A}$ service provision in 'Containment \& Buffer Zone' and 'areas beyond Buffer Zone \& Green Zone'. The Guideline has recommended facility based immunization service delivery to walk-in beneficiaries on demand basis in containment and buffer zone. There will be no outreach session and active mobilization in such area. However, in area beyond Buffer Zone \& Green Zone, facility based immunization and outreach sessions can be conducted with modified health, sanitation and nutrition day (VHSND/UHSND), either by adopting staggered approach (hourly slots \& alternate session sites) and/or by adopting break-up session to avoid crowding. The guideline 
has suggested continuation of the birth dose of vaccine at all health facilities irrespective of zones because beneficiaries will be in the health facility only.

Even though, the guideline has provided much needed guidance and support to the States/UTs in carrying-out immunization activities but dynamicity due to change in number of COVID-19 cases as well as shift in zone status from containment to buffer/green zone or viceversa may require frequent changes in session planning \& implementation, IEC activities, capacity building of HR and beneficiary tracking exercise.

\section{Use of Digital Platform for Tracking of Beneficiaries and Awareness Generation}

The headcount survey conducted by Field Level Workers (FLWs) and due-list prepared by them before lockdown may not reflect the actual number of beneficiary in the catchment area mainly due to missed vaccination, new birth cohort and entry of migrants population from other parts of the country, during the time elapsed. In addition, non-availability of Mother and Child Protection (MCP) card especially among migrant workers who were the part of mass movement occurred during COVID-19 may increase difficulty of field level workers while tracking the immunization history of the beneficiary. If immunization history is not properly recalled by the caregiver, it may lead to duplication or missed vaccination. The chances of Adverse Event Following Immunization (AEFI) may not be ignored in such situation. The delayed immunization and/ or incorrect immunization history of beneficiaries may also affect the estimates to be done by FLWs regarding new beneficiaries \& missed children. They may face several questions for example, if a child had late vaccination and missed doses, is vaccine to be restarted in case of doubtful immunization history or to be resumed without repeating vaccines given.

Aadhar linked Electronic Immunization Registry (EIR) application may be considered for a registry of vaccinated children, adolescent and pregnant women. The application may use aadhar number of parents \& pregnant women for registration using beneficiary name, date of birth, place of delivery and immunization event. Application may be a separate android\& iOS based application or as a part of ANMOL (ANM Online) application. The EIR may help in sending messages regarding antigen given and due date for the next antigen, session details and other important messages regarding COVID-19 triage.

The UIDAI Guideline titled UIDAI Strategy Overview, 2010 had also suggested linking of UIDAI number with birth certificates for immunization services. ${ }^{13}$ Further, a guideline on "Electronic Immunization Registry: Practical Considerations for Planning, Development, Implementation, and Evaluation" of Pan American Health Organization 2017 has discussed EIR process and various case studies about successful use of EIR in Uruguay, Peru and Bogota, Colombia etc. The guideline mentions that in Bogota, EIR facilitates data analysis and generation of outreach indicators by place of residence or immunization, as well as of vaccination by month of birth (cohort monitoring); supports rapid monitoring of coverage and calculation of dropout rates; and enables identification of children with incomplete immunization schedules". ${ }^{14}$ Therefore, these global experience may be considered to develop and implement effective "EIR" system in India.

Use of digital technology in awareness generation among staff and parents is an effective way to disseminate the credible and updated information. IEC materials for FLWs, health managers and caregivers may be provided through online media (whatsapp, youtube, facebook, twitter) well in advance.

\section{Asymptomatic SARS-CoV-2 Infection in Children}

As observed worldwide, several asymptomatic cases of COVID-19 disease are there. Likewise, children with COVID-19 may not show sign \& symptoms such as fever and cough ${ }^{15}$ thus it will be a challenging task for vaccinator to have an appropriate suspicion of coronavirus but also to check for other problems like flu and plan immunization of such children later or separately as per the social distancing protocol of the Gol. As children are more prone to infection, all necessary precaution must be taken by FLWs. Staff need to be trained in differentiating COVID-19 \& common flu, procedure for covering children of quarantine families, referral \& surveillance process for COVID-19 cases etc. either through online mechanism or offline in small batches and after following required precautions.

\section{Likelihood of VPD Outbreak Due to Prolonged Disruption of Immunization Services}

In National Immunization Schedule, a gap of at least 4 week (28 days) is provisioned between two doses of vaccine but immunization services are generally defined as delayed if immunization is not given for more than one month. Disruption of immunization services for long-term, may lead to likelihood of VPD outbreak especially in case of measles, diphtheria and wild poliovirus (type-2).

- Measles cases are more liable to be increased because of prolonged stagnation of immunization services. Various studies found that gastrointestinal and respiratory complications of measles were common and increased mortality \& neurology complications were associated with malnutrition. ${ }^{16}$ The COVID-19 situation is also adversely affecting nutrition and other essential health services that can directly or indirectly result in VPD outbreak and high rate of morbidity and mortality. 
Prolonged delay of SIA may increase the likelihood of measles outbreak as witnessed in African countries during EBOLA outbreak (2014-15). ${ }^{17}$ Campaign/mass vaccination, if not planned well during the pandemic after adopting required precautionary measures, we may not be able to cover missed children and large birth cohort though RI only.

- India has been declared polio-free in 2014 but transmission of wild poliovirus (type-2) between two countries may cause outbreak if not preplanned well during COVID pandemic. Under UIP, the Inactivated Polio Vaccine (IPV) is being used to boost immunity among children who has received birth dose of Oral Poliovirus Vaccine (OPV) to ensure interruption of any residual polio virus transmission but global experience of IPV supply and pricing issues, if not tackled during pandemic, may navigate to polio outbreak situation. The possibility of importation of the polio strain from another State/UTs or Country after lockdown need to be duly considered.

- The summer season may also bring the possibility of occurrence of Japanese Encephalitis and Acute Encephalitis Syndrome (AES) in endemic districts, therefore, adequate planning and availability of JE vaccine (mostly imported from China as well as indigenously developed) is need of the hour.

- In 2019, several districts in India reported diphtheria outbreak. Therefore, based on the learning of the recent outbreak surveillance and management, a suitable strategy may be devised. If any resurgence of diphtheria happen in previous and/or new pockets due to missed vaccination as well as declining immunity, it will create and additional burden on already overburdened health system.

\section{Strengthen Health Human Resources/FLWs to Deliver Immunization and COVID- I 9 Services}

Health Human Resources including Auxiliary Nurse Midwifery (ANM), ASHA and Anganwadi Workers (AWW) have been diverted towards management of COVID-19 pandemic. Ground level presence of development partners is also limited. Therefore, there are high chances that overwhelmed public health system may likely face direct losses due to rising cases \& death from COVID-19, and indirect morbidity and mortality from Vaccine Preventable Disease (VPD) outbreak, if not managed well in timely manner. District may need to plan deployment and ensure optimal utilization of HR, for immunization service delivery as well. FLWs must be equipped with personal protective equipment such as three layered mask, apron, gloves, sanitizers, hand wash, full face protection shield etc. Use of sanitization after each immunization, maintaining social distancing norm must be followed strictly.
Use of Aarogya Setu app may be extensively promoted among all beneficiaries \& health staff. ${ }^{18}$ Creating a virtual call id (such as zoom, whatsapp, google meeting) for coronavirus positive individual of the area may enable the FLWs to interact with patients having minor symptoms without risk exposures. Use of telemedicine by health staff may also help in questionnaire based rapid screening and to triage patients at home or hospital based on the screening result. These FLWs can be involved in immunization services as well with all precautionary measures as per SOP for COVID-19 management. However, considering the children's immune power, staff involved in immunization services may be exempted from managing COVID-19 cases by in-person visit. ${ }^{19}$

\section{Adherence to Standard Operating Procedure for COVID- 19 Management}

The Standard Operating Procedure (SOP) for COVID-19 management released by Gol suggested three standard precautions - hand hygiene, use of barriers precautions or Personal Protective Equipment (PPE) and safe injection practices that need to be followed strictly. However, ensuring availability and use of PPEs by all service delivery staff, formulation of hand washing facilities at immunization fixed session sites and cold chain room, and provision of alcohol based hand rub/sanitizer to FLWs for perusal during outreach sessions may be one of the biggest challenges before resumption of immunization services.

\section{Sustaining VPD Surveillance System}

Along with immunization sessions, VPD surveillance activities if suspended or limited, need to be revoked as soon as possible. Even though many laboratories and sample collection facilities are engaged in COVID-19 related testing and investigations, VPD surveillance activities may require to be continued for detecting VPD outbreak and responding to it quickly to prevent further spread of VPDs and to understand vaccine effectiveness and safety overtime. ${ }^{20}$

\section{Role of Parents in getting their Children Immunized}

The involvement of parent \& caregiver are very important. Parent's trust on UIP \& public health services, awareness about VPDs and UIP schedule, understanding about importance of immunization, literacy \& employment status, attitudes and beliefs plays critical role in receiving immunization services. Therefore, FLWs must adopt evidence based approach and behavior change communication strategies to create awareness among the parents and caregivers and ensure their involvement.

In this uncertain scenario during COVID-19, parents/ caregivers may have several questions in mind.

- How to ensure child immunization in COVID-19 zones? 
- Where to go for immunization services - fixed or outreach?

- Is immunization safe during COVID-19 even at facility?

- What will be the possible cons if child does not receives immunization as per schedule

Therefore, an informative advisory and/or IEC materials in local language may be developed and shared through whatsapp, Facebook, TV, YouTube and other audio-visual media. This may help FLWs in addressing concerns of parents, creating awareness, and ensuring community support during the pandemic.

\section{Community Mobilization, Communication and IEC}

ASHA and AWW are accountable for mobilizing community for immunization services, however, reports of attack on health workers engaged in COVID-19 screening \& followup may have built an unseen barrier between ASHA/AWW and community and will adversely affect the mobilization part of immunization services. Even after resumption of immunization in well planned manner, majority of caregivers may believe that keeping children indoor will keep them safe from COVID-19. Therefore, effective IEC activities communicating risk of not completing the immunization and COVID-19 safety process must be conducted. A plan of action for regaining and maintaining public confidence in immunization services during COVID-19 is critical to ensure that each and every child, adolescents and pregnant women are covered. It is also needed to ensure that due vaccination to be provided to restore declining trend in vaccine coverage rate and to combat the likelihood of VPD outbreak due to prolonged suspension/missing vaccination. Non-availability or shortage of reporting formats, MCP cards, IEC materials etc. may be witnessed due to closure/limited functioning of printing press. Transportation and distribution of these formats from states headquarters to districts and then to sub-center level may also be critical task during present scenario. Use of digital technology may be an effective way to deal with such issues during pandemic situation.

\section{Other Issues}

The global pandemic may affect the immunization programme by leading to, decline in financial, HR and other resources, social-instability, limiting political attention and Country's health priority. It may affect the vaccine production to its dissemination plan as there would be no entry of new vaccine manufactures, limited vaccine production capacity globally, increase costing, failure of market shaping strategies for price negotiation, high freight charges, inadequate vaccine availability, infrequent vaccine supply and distribution. In order to deal with such situation, if faced, innovative strategies may be developed indicating opportunity of alternate option so that the situation does not adversely impact immunization services.

\section{Conclusion}

The article may guide the program managers at the national, state and district level while planning a resumption of immunization services during COVID-19 pandemic and thereafter. The article will act as a tool that will support in planning, implementation, and monitoring of immunization services in the pandemic situation and; may promote further strengthening of immunization system in the country in emergency context. This paper may also help other countries which are planning to resume immunization services and may support them in devising strategies and development of an action plan.

\section{Recommendation/ Suggestions}

- Resumption of routine immunization service as soon as possible, considering missed immunization during COVID-19 lockdown and likelihood of VPD outbreaks (measles, diphtheria \& Polio-type-2), if there is prolonged delay in vaccination.

- Instead of 'One-Size-Fits-All' strategy, a 'Tailored based approach' may be followed for containment \& buffer zone, beyond buffer zone and green zone. The districts/block Immunization Task Force may review the existing situation of COVID-19 in the respective areas and suggest session planning accordingly considering COVID-19 containment measures and Gol guidelines.

- COVID-19 testing of all staff involved in immunization services such as FLWs, cold chain handlers, supervisors \& Alternate Vaccine Delivery (AVD) may be done before resumption of immunization services to detect asymptomatic infection in healthcare workers, if any. If not considered, it may inadvertently cause transmission of COVID-19 to fellow health workers, children, pregnant women and caregiver.

- District/block health staffs to identify safe and well ventilated session site, ensure adequate availability and use of PPE and hand hygiene measures by FLWs and caregivers, adopt social distancing by limiting number of caregivers and/or use of token system/queue.

- $\quad$ FLWs need to conduct headcount survey and prepare due list to include new beneficiary (new births and migrants) and missed children during lockdown. Number of revised beneficiary as per due list/microplan will help in estimation of vaccine and logistics requirement at the session site.

- Number of RI days may be increased or immunization may suitably be done in many shift to avoid gathering at a time. Immunization at fixed session site may be promoted. If urgent, mass vaccination may be allowed after reviewing the COVID-19 situation in area and strictly adopting all preventives measures for COVID-19. Focus to be continued on VPD surveillance system to keep watch on VPDs outbreak, if any, and timely action 
to mitigate the risk.

- $\quad$ Suitable provisions for paperless recording, reporting and IEC may be required in case of non-availability/ shortage of reporting formats, MCP cards, IEC materials etc.

- For community mobilization and monitoring, use of mobile calls or messages may be promoted. If needed, only assigned supervisor or staff of supporting agencies may be allowed to visit session sites for monitoring and support.

- Involvement of religious and political leaders, media and local influential persons, especially in areas where community is against COVID-19 testing, may ensure safety, security and much needed support to healthcare workers during field activity.

- There may be possible chances of community refusal/ vaccine hesitancy and violence against FLWs by unsocial elements during COVID-19 and thereafter as well. Therefore, appropriate safety measures for FLWs including district/block helpline number, immediate response from police may need to be considered.

- Demand-supply analysis of vaccine and logistics may be carried out based on the revised estimates of missed children and new birth cohorts. Considering travel restriction (both international \& national) bulk procurement of vaccine and logistics may need to be done. Therefore, assessment of cold chain space for bulk supply and keeping buffer stock may be required as soon as possible. However, these activities are directly linked with revised estimated of beneficiaries in states/ districts.

- $\quad$ Considering sudden hike in global vaccine requirement at a time, limited vaccine production capacity, no new entry of WHO prequalified vaccine manufacturer, diverted research \& production capacity for COVID-19 vaccine, possibility of increased costing due to failure of market shaping strategies for price negotiation, high freight charges, global travel restrictions etc.; India may need to explore indigenous manufacturers and/ or suitable alternate options to ensure uninterrupted vaccine and logistics availability during pandemic and in future as well.

\section{Conflict of Interest: None}

\section{References}

1. Coronavirus Pandemic Update (2020, June 6), Retrieved from World Metersinfo: Available from: https://www. worldometers.info/coronavirus/.

2. Press Release, Ministry of Home Affairs, Extension of Lockdown for a further period of two weeks with effect from 4 May 2020, Posted on: 01 May 2020 on 6:33PM by PIB Delhi available from: https://pib.gov. in/PressReleasePage.aspx?PRID=1620095.
3. Universal Immunization Program guideline, Ministry of Health and Family Welfare. Available from: https://main. mohfw.gov.in/sites/default/files/5628564789562315. pdf.

4. Press Release, Ministry of Finance, Samagra Siksha 2018-19, Posted on: 31 Jan 2020 on 1:15PM by PIB Delhi. Available from: https://pib.gov.in/PressReleasePage. aspx?PRID=1601247.

5. IMI-Coverage Evaluation Survey Report (2018), Ministry of Health and Family Welfare, Government of India.

6. Press Release, Ministry of Health and Family Welfare, Intensified Mission Indradhanush,Posted on: 7 Feb 2020 on 12:55 PM by PIB Delhi. Available from: https:// pib.gov.in/newsite/PrintRelease. aspx?relid=199090.

7. National Vaccine Policy, Ministry of Health and Family Welfare. Available from: https://main.mohfw.gov.in/ sites/default/files/108481119000.pdf.

8. Comprehensive Multiyear Plan (2018-22) for Immunization, Ministry of Health and Family Welfare. Available from: https://nhm.gov.in/New Updates_2018/NHM_Components/Immunization/ Guildelines_for_immunization/cMYP_2018-22_final_ pdf.

9. GAVI-India Joint Appraisal Report, 2019, GAVI- The Vaccine Alliance. Available from: https://www.gavi.org/ sites/default/files/document/2020/India\%20Joint\%20 Appraisal\%20Report\%202019.pdf.

10. India's proposal for HSS support, 2017. GAVI-The Vaccine Alliance. Available from: https://www.gavi. org/sites/default/files/document/proposal-for-hsssupport-2017--indiapdf.pdf.

11. National Factsheet, Coverage Evaluation Survey 2009, UNICEF.

12. Guideline on Enabling Delivery of Essential Health Services during the COVID-19 Outbreak. Posted on 14 April 2020. Available from: https://www.mohfw.gov.in/ pdf/.GuidanceNoteonProvisionofessentialRMNCAHNServices24052020.pdf.

13. UIDAI Strategy Overview-Creating A Unique Identity Number For Every Resident in India, Planning Commission, Posted on: April 2010. Available from: https:// www.prsindia.org/sites/default/files/bill_files/UIDAI_STRATEGY_OVERVIEW.pdf.

14. Electronic Immunization Registry: Practical Considerations for Planning, Development, Implementation, and Evaluation", WHO and Pan American Health Organization 2017. Available from: https://iris. paho.org/bitstream/handle/10665.2/34865/9789275119532_eng. pdf? sequence $=5$ \&isAllowed $=y$.

15. Information for Pediatric Healthcare Providers, Center for Disease Control (CDC), Posted on: 3 May 2020. Available from: https://www.cdc.gov/coronavirus/2019-ncov/hcp/pediatric-hcp.html. 
16. Eugene Lam, Amanda McCarthy, and Muireann Brennan, Vaccine-preventable diseases in humanitarian emergencies among refugee and internally-displaced populations, 2015. Available from: https://www.ncbi. nlm.nih.gov/pmc/articles/PMC4685677/.

17. Balcha Girma Masresha, Richard Luce Jr, Goitom Weldegebriel, Reggis Katsande, Alex Gasasira, Richard Mihigo. The impact of a prolonged ebola outbreak on measles elimination activities in Guinea, Liberia and Sierra Leone, 2014-2015, Posted on Jan 2020: Available from: https://www.ncbi.nlm.nih.gov/pubmed/32373259.

18. Press Release, Ministry of Electronics \& IT, government of India launches Arogya Setu App to track COVID-19 infection, Posted on: 02 April 2020 on 4:58PM by PIB Delhi. Available from: https://pib.gov.in/PressReleaselframePage.aspx?PRID=1610326.

19. Kapoor A, Gupta S, Yadav R. Digital healthcare: The only solution for better healthcare during COVID-19 pandemic? Available from: https://www.researchgate.net/publication/340587165_Digital_Healthcare_ The_only_solution_for_better_healthcare_during_ COVID-19_pandemic

20. CDC Washington, U.S. Public Health Response to the Measles Outbreak, 2019, Posted on 27 Feb 2019. Available from: https://www.cdc.gov/washington/ testimony/2019/t20190227.htm. 